\title{
Development of Microcapsules
}

\author{
Brúnó Vermes ${ }^{1,}$ a $^{*}$, Tibor Czigány ${ }^{1,2, b}$ \\ ${ }^{1}$ Department of Polymer Engineering, Faculty of Mechanical Engineering, Budapest University \\ of Technology and Economics, Múegyetem rkp. 3., H-1111 Budapest, Hungary \\ ${ }^{2}$ MTA-BME Research Group for Composite Science and Technology, Műegyetem rkp. 3., \\ $\mathrm{H}-1111$ Budapest, Hungary \\ avermesbruno@gmail.com, bczigany@eik.bme.hu \\ ${ }^{*}$ corresponding author
}

Keywords: microcapsules, PETMP hardener, melamine-formaldehyde, colored filling

\begin{abstract}
Melamine-formaldehyde (PMF) coated microcapsules were produced with liquid pentaerythritol-tetrakis(3-merkaptopropionate) (PETMP) and UV-sensitive indicator colored PETMP cores. Capsules with an average diameter of $43 \mu \mathrm{m}$ formed due to the adjustments on magnetic stirring, compound ratios, acidity and reaction time. Reproducible recipe was found to produce colored PETMP filled capsules with yields of 1-10\%. Optical microscopic investigations were conducted to follow the formation of the particles, and scanning electron-microscope (SEM) was used to verify that the capsules did not break up as an effect of the brittle failure of the epoxy matrix specimens. Improvement of the shell-matrix adhesion was attempted with silane compounds and investigated with X-ray photoelectron spectroscopy (XPS) and SEM.
\end{abstract}

\section{Introduction}

Polymers and composites with microencapsulated agents can be used as self-marking, or either self-healing systems if the micro-sized particles fulfill some basic requirements. Some of these are the appropriate relative thickness of the solid, polymer shell, and the needed quality and relative quantity of the capsules` liquid filling [1].

Successful experiments have shown that it is possible to produce capsules with dicyclopentadiene [2-4], epoxy and different kinds of epoxy-hardener [5-7] cores besides some others, filled with less frequently investigated agents. The solid shells are most commonly made of urea-formaldehyde [8-10] or PMF [10-12] as these substances meet all the necessary requirements in most cases. Much emphasis is on the self-healing ability of polymers [13] and even polymer composites [14] involving two components, while the possibility of self-marking [15] is less pronounced in literature. While one of the components has to be encapsulated for isolation purposes, the other one does not have to be so, thus it can be dispersed in the matrix or on the surface of the capsules [16]. Chemical reaction of healing starts only through the crack of the capsule as the compounds mix.

The rupture of a capsule occurs only if the crack inside the matrix is able to deliver the needed proportion of its energy onto the solid shell. The required energy depends on the size and the relative measurements of the capsules, and even the adhesion between the shell and the matrix. If physical adsorption is not sufficient, it is feasible to join them chemically to improve adhesion [17].

Prior results [18] gave the base of the work, but could not provide the repeatability. This paper aims to put down a reproducible recipe of manufacturing PETMP and UV-sensitive indicator colored PETMP cored capsules with PMF shells. Modifying parameters of the literal recipe was necessitated to achieve the same quality of hardener filled capsules (colored and uncolored ones) throughout the repeated experiments. In addition, creation of chemical bonding was attempted in order to improve the strength of the epoxy matrix-shell adhesion. 


\section{Materials and methods}

Materials. Purified, distilled water was obtained from the Department of Organic Chemistry and Technology (Budapest University of Technology and Economics). Melamine (99\%), triethanolamine, Keystone rhodamine B base (95\%), ethylene maleic anhydride (EMA) and the needed silane compounds (see below) were purchased from Sigma-Aldrich. Formaldehyde (37\%, stabilized with methanol) was obtained from Merck Hungary, while all the used equipment (flasks, filters, etc.) were bought in AA-labor (Hungary).

Preparation of microcapsules. In situ polymerization in an oil-in-water emulsion resulted the filled balls during magnetic agitation. The main steps of the process were the creation of the dyed hardener drops in centrifugal force field, the migration of the shell-material onto the core-drops and its polymerization on the surface.

Microcapsule formation and size analysis. Samples were taken during the experiments and investigated with optical microscope (Olympus BX 51M), digital camera (C-5060 CAMEDIA) and image analysis software (AnalySIS). Average diameter and standard deviation were determined in the case of the dyed capsules from data of 100 measurements.

Embedding. Capsules were dispersed in a 3:1 mixture of epoxy (ipox MR 3012) and hardener (ipox MH 3122). Specimens were fabricated with $0.5 \%$ capsule content and were broken with a rate of $2500 \mathrm{~mm} / \mathrm{min}$ in order to achieve brittle failure.

Scanning electron microscopy. After brittle failure, breaking surfaces of specimens with embedded microcapsules in epoxy matrix were investigated with SEM (JEOL JSM 6380LA). Samples were sputtered a thin layer of gold in order to prevent charging under electron beam.

Surface treatment. Prior surface treatment was attempted on the capsules before the dispersion in order to improve the subsequent shell-matrix adhesion. $0.5 \mathrm{~g}$ microcapsule was agitated slightly in a mixture of $50 \mathrm{ml}$ buthanol and $0.05 \mathrm{~g}$ silane compound ((3-Aminopropyl)triethoxysilane / (3Glycidoxypropyl)trimethoxysilane) for 2 hours, then rested at room temperature for 3 days. After distilling the excess solvent from the capsules, they were put into $100 \%$ vapor for another 3 days and then dried.

Elemental analysis. MultiQuant 7.50 XPS device was used to find out if there was any silane (silicium) left on the surface of capsules after the surface treatment.

\section{Results and discussion}

Reproducible recipe. Increasing the intensity of agitation, the size of the drops decreased, while longer time of the reaction caused thicker solid shells. In case of too high, $\mathrm{pH}>10$ values in the prior mixture of the shell-material, continuous solid PMF could not develop, or - slightly above $\mathrm{pH} 10$ foam-like structures were created as the polymerization was not quick enough to result individual capsules. At lower $\mathrm{pH}$ values $(\mathrm{pH}<10)$ aggregates were formed of PMF particles as the reaction was too quick for the shell-material to migrate onto the surface of the 'core' droplets and instead it formed separate growths. Finding the optimal acidity meant the mean of these two borderlines, and resulted individual capsules without any aggregates. Illustrative examples are shown in Figure 1 for each case.
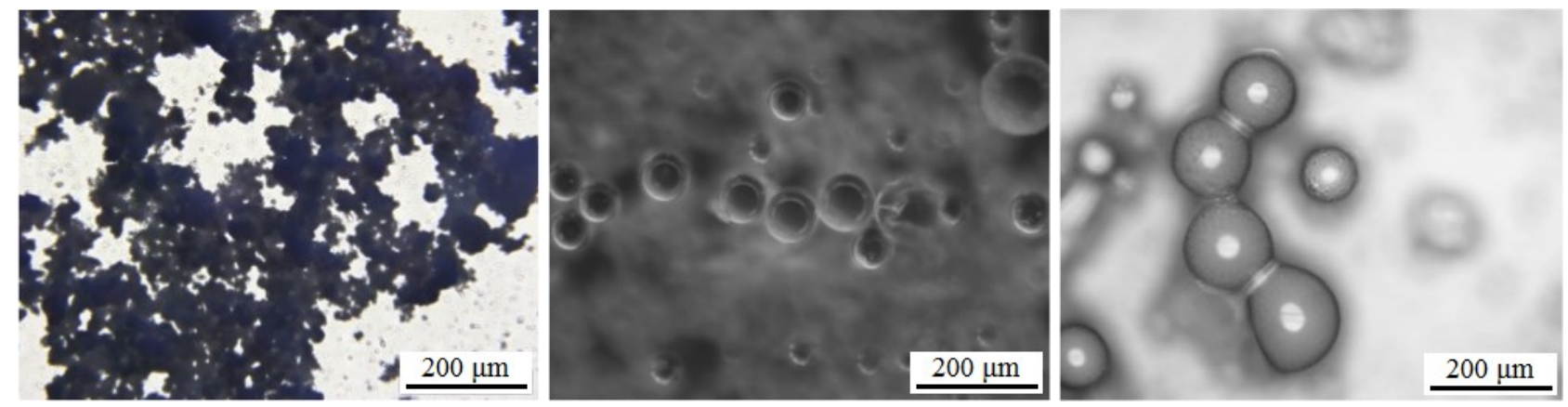

Figure 1. Aggregates at $\mathrm{pH}<10$, capsules at $\mathrm{pH}=10$ and foam-like structures at $\mathrm{pH}>10$ 
Considering the results of the studied literature and as a result of a thorough optimization, the right parameters were found for producing the pure and dyed hardener cored capsules.

At room temperature $\left(20-22^{\circ} \mathrm{C}\right), 12 \mathrm{ml}$ of $2 \%$ previously prepared EMA solution was mixed with $2.5 \mathrm{~g}$ PETMP and $0.05 \mathrm{~g}$ indicator dye (rhodamine B) in a $50 \mathrm{ml}$ round bottomed flask. At $70^{\circ} \mathrm{C}, 0.625 \mathrm{~g}$ melamine, $1.335 \mathrm{~g}$ of $37 \%$ formaldehyde, $1 \mathrm{ml}$ distilled water and $0.2 \mathrm{ml}$ (4 drops) of triethanolamine were heated for 10 minutes during slight agitation. The latter mixture was poured into the other one, and $1^{\circ} \mathrm{C} /$ minute heating was applied up to $65^{\circ} \mathrm{C}$ to provide the thermal condition of polymerization. After 2 hours of reaction, the mixture was filtered and rinsed with acetone on a glass filter (10-16 $\mu \mathrm{m})$ using small scale vacuum. In figure 2, a flowchart of the process can be seen with the necessary data.

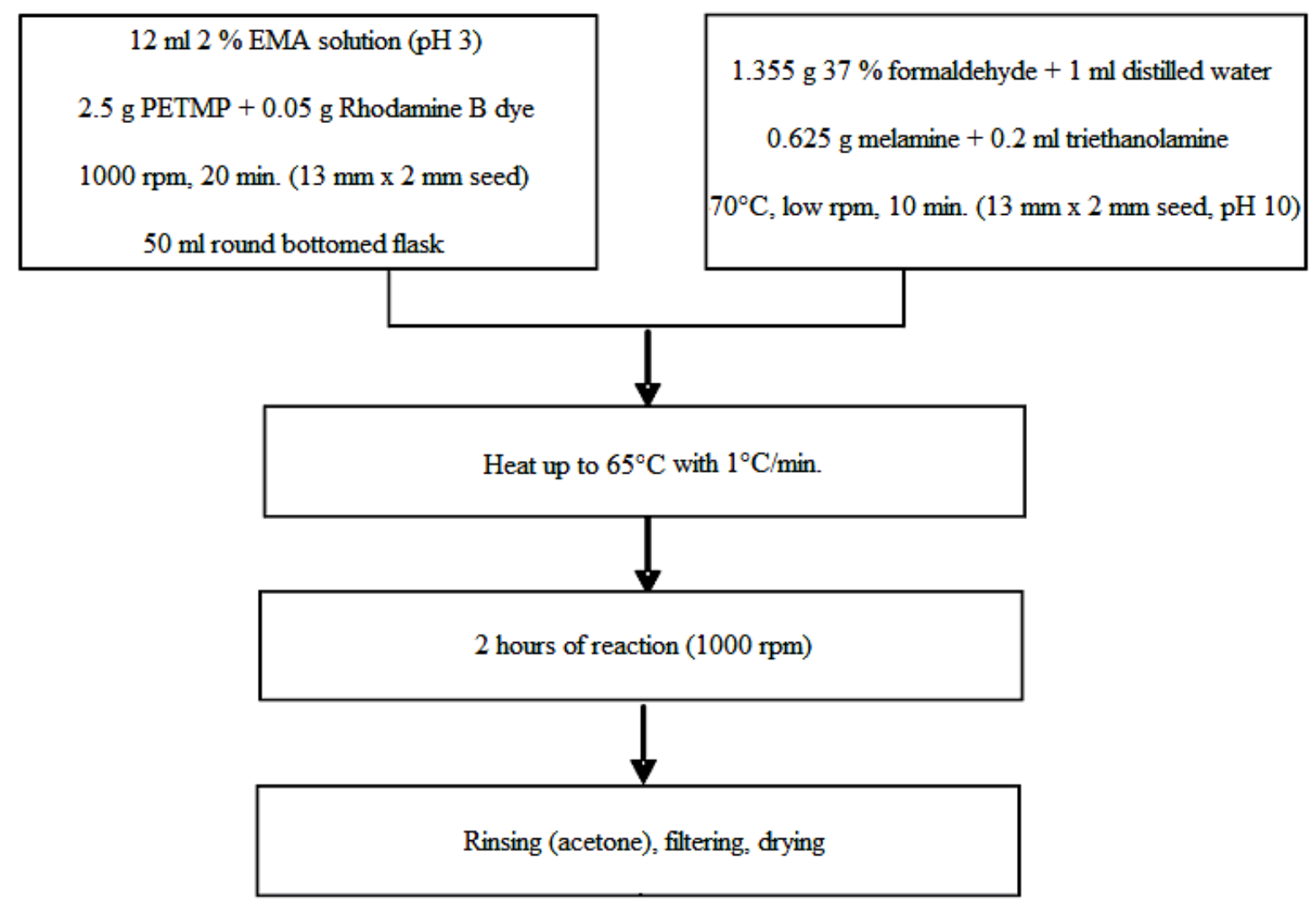

Figure 2. Dyed PETMP encapsulation recipe

Yield and diameter. After the rinsing and filtering steps, the dried product - which contained only microcapsules without any significant contaminants - weighted the $1-10 \%$ of the theoretically calculated maximum. However the main reason of the loss was the difficulty of filtering, not the encapsulation process itself. Optical measurements showed an average diameter of $43 \mu \mathrm{m}$ with a standard deviation of $12 \mu \mathrm{m}$.

Fill content. Having some of the capsules crushed between glass plates, the liquid core filled the space between the shells (Fig.3). The possibility of future applications was provisioned by judgement from the quantity of the stored component.

A small amount of the core material was put under UV-lamp in a dark room. Intense pink/purple light proved that the indicator property of the dye remained unchanged. Calculated amount of epoxy resin hardened after mixing it with the core material, giving the core material all the properties that were required in terms of both quantity and quality.

Shell-matrix adhesion. Studying the SEM images of the epoxy-matrix composites' breaking surface, it seems obvious that the adhesion was not great enough between the capsule and the matrix. Undamaged capsules stuck out from the surface (Fig. 3) as the crack was not able to deliver the sufficient proportion of its energy onto the shells for rupture, and instead it by-passed them. 
XPS results showed that the attempt of layering silane compounds on the shells was successful, however after investigating the breaking surface of the specimens with the improved capsules, the SEM results were similar to the previous case in terms of the undamaged shells. Surface boundary layer formation of the silane without any chemical bonding to the shell is the most probable explanation for the results.

As certain functional groups of the PMF shell (amine) and the epoxy matrix (epoxy) are able to react with each other and establish covalent bonds without any prior surface treatment. The direction of the future experiments is to provide the needed thermal conditions for the reaction, and so improve the adhesion.
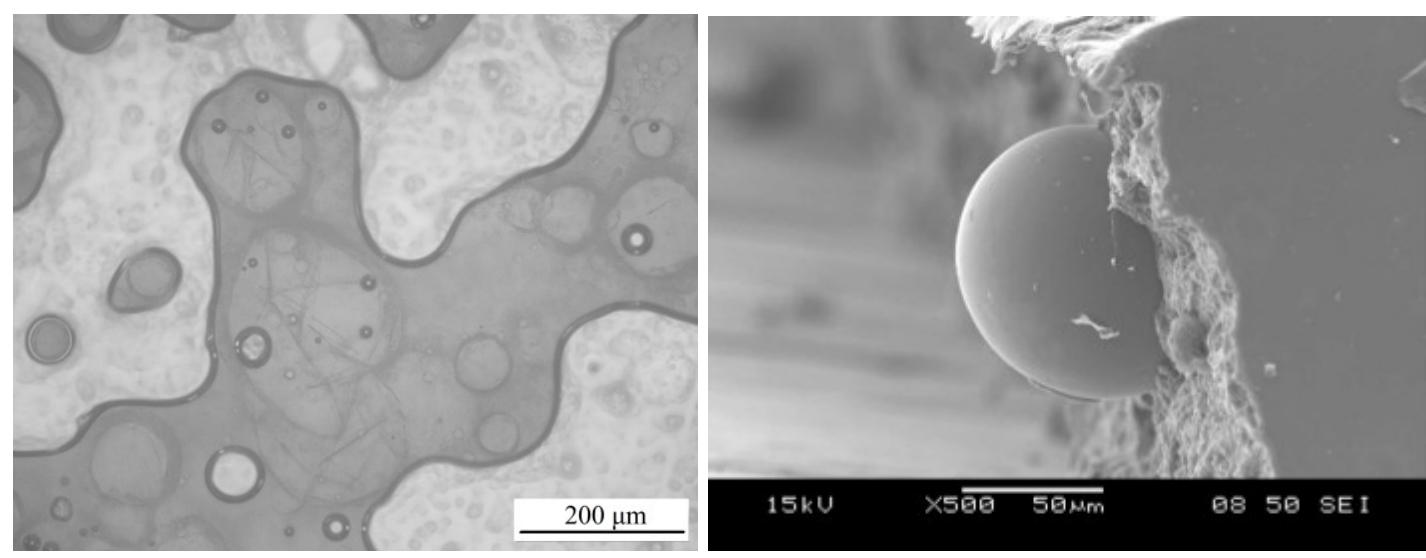

Figure 3. Liquid core material between the crushed shells (left) and SEM image of an undamaged microcapsule sticking out from the breaking surface (right)

\section{Conclusion}

A reproducible process for encapsulation PETMP and UV-sensitive dye colored PETMP by in situ polymerization of melamine-formaldehyde (PMF) was developed by following the effects of the changed parameters optically (microscope). An average diameter of $43 \mu \mathrm{m}$ was measured besides $1-10 \%$ yields which was a result of the separation difficulties. Microcapsule core material remained unchanged during the process in terms of its functionality, thus its quality and quantity provide the possibility of application of the capsules in the future. Scattering the capsules in epoxy resin, specimens were produced, and their breaking surfaces were investigated with SEM after brittle failure. As the capsules remained intact, their prior surface treatment was attempted with silane compounds. However their migration to the surface of the shells was successful, SEM images similar to the previous case indicated that the adhesion did not increase significantly, presumably because of the lack of chemical bonding. To improve the capsule-matrix adhesion, surface reaction of the shells and resin will be attempted in the future by providing the required thermal conditions for the reaction.

\section{Acknowledgement}

This paper was supported by National Research, Development and Innovation Office - NKFIH, OTKA K 116070. 


\section{References}

[1] S.K. Ghosh, Self-healing Materials: Fundamentals, design strategies, and applications, in: S.K. Gosh, Self-healing Materials, 6, Wiley-VHC, Weinheim, Germany, 2009, pp. 1-25.

[2] S.R. White, N.R. Sottos, P.H. Geubelle, J.S. Moore, M.R. Kessler, S.R. Sriram, E.N. Brown, S. Viswanathan, Autonomic healing of polymer composites, Nature, 409 (2001) 794-797.

[3] S.R. White, N.R. Sottos, P.H. Geubelle, J.S. Moore, S.R. Sriram, M.R. Kessler, E.N. Brown, US Patent: 6,518,330-B2. (2003)

[4] B.J. Blaiszik, N.R. Sottos, S.R. White, Nanocapsules for self-healing materials. Composites Science and Technology, 68 (2008) 978-986.

[5] T. Yin, M.Z. Rong, M.Q. Zhang, G.C. Yang, Self-healing epoxy composites - Preparation and effect of the healant consisting of microencapsulated epoxy and latent curing agent, Composites Science and Technology, 67 (2007) 201-212.

[6] H. Jin, C.L. Mangun, D.L. Stradley, J.S. Moore, N.R. Sottos, S.R. White, Self-healing thermoset using encapsulated epoxy-amine healing chemistry, Polymer, 53 (2012) 581-587.

[7] Y.C. Yuan, M.Z. Rong, M.Q. Zhang, Preparation and characterization of microencapsulated polythiol, Polymer, 49 (2008) 2531-2541.

[8] E.N. Brown, M.R. Kessler, N.R. Sottos, S.R. White, In situ poly(urea-formaldehyde) microencapsulation of dicyclopentadiene, Journal of Microencapsulation, 20 (2003) 719-730.

[9] H. Li, R. Wang, H. Hu, W. Liu, Surface modification of self-healing poly(urea-formaldehyde) microcapsules using silane-coupling agent, Applied Surface Science, 255 (2008) 1894-1900.

[10] Y. Konuklu, H.O. Paksoy, M. Unal, S. Konuklu, Microencapsulation of fatty acid with Poly(melamine-urea-formaldehyde), Energy Conversion and Management, 80 (2014) 382-390.

[11] L. Yuan, G.-Z. Liang, J.-Q. Lie, Synthesis and characterization of microencapsulated dicyclopentadiene with melamine-formaldehyde resins, Colloid Polymer Sci., 285 (2007) 781-791.

[12] M.Q. Zhang, M.Z. Rong, Self-healing polymers and polymer composites, Wiley-VHC, Weinheim, Germany, 2011.

[13] M.U. Saeed, B.B. Li, Z.F. Chen, S. Cui, Self-healing of low-velocity impact and mode-I delamination damage in polymer composites via microchannels, Express Polymer Letters, 10 (2016) 337-348.

[14] M.Q. Zhang, Polymer self-heals in seconds, Express Polymer Letters, 9 (2015) 84-84.

[15] W. Li, J.W. Buhrow, S.I. Jolley, L.M. Calle, J.S. Hanna, J.W. Rawlins, Microencapsulation of corrosion indicators for smart coatings, DoD Corrosion Conference, Cocoa Beach, Florida, United States (2011) ID: 20110014386

[16] A.F. Skipor, S.M. Scheifer, B. Olson, US Patent: 0,007,784 -A1. (2004)

[17] Y. Xie, C.A.S. Hill, Z. Xiao, H. Militz, C. Mai, Silane coupling agents used for natural fiber/polymer composites: A review, Composites: Part A, 41 (2010) 806-819.

[18] A. Czeller, T. Czigány, Effect of healing agent-loaded microcapsules on the mechanical properties of self-healing epoxy composites, ECCM15 - 15th European Conference on Composite Materials, Venice, Italy (2012) 1353. 